\title{
Formative Assessment of ARM-U: A Modular Intervention for Decreasing Risk Behaviors Among HIV-Positive and HIV-Negative Methamphetamine- Using MSM
}

\author{
Richard S. Garfein ${ }^{*}, 1$, Mitcheal Metzner ${ }^{1}$, Jazmine Cuevas ${ }^{1}$, Chad A. Bousman ${ }^{2}$ and \\ Thomas Patterson ${ }^{2,3}$ \\ ${ }^{I}$ Division of Global Public Health, Department of Medicine, University of California, San Diego, La Jolla, California, \\ USA \\ ${ }^{2}$ Department of Psychiatry, University of California, San Diego, La Jolla, California, USA \\ ${ }^{3}$ Department of Veteran's Affairs Medical Center, San Diego, La Jolla, California, USA
}

\begin{abstract}
Background: Methamphetamine is a major contributor to HIV transmission among men who have sex with men (MSM). Recent studies show that up to one-third of methamphetamine-using MSM (MUMSM) inject the drug. We developed a behavioral intervention for MUMSM to decrease unprotected anal intercourse and increase awareness of parenteral HIV transmission risk. This 6 -session ( 3 in-person, 3 by telephone) modular intervention was designed to be tailored to participants' HIV (+/-) and injection drug user ([IDU] yes/no) status. We present results of formative research used to evaluate the content and to assess feasibility and acceptability of this individual-level HIV risk-reduction intervention.
\end{abstract}

Setting: HIV research clinic in a high MSM and methamphetamine prevalence neighborhood.

Project: Avoiding Risks from Methamphetamine-Use (ARM-U) is a brief toolbox intervention that allows counselors to select modules that suit a client's individual risk profile and intervention needs employing motivational interviewing and cognitive behavioral theory. We evaluated the format and content of the intervention through focus groups and pre-testing of the entire intervention using volunteers from the target population stratified into four groups (HIV+/IDU, HIV-/IDU, $\mathrm{HIV+/non-IDU,} \mathrm{HIV-/non-IDU).} \mathrm{Four} \mathrm{individuals} \mathrm{in} \mathrm{each} \mathrm{stratum} \mathrm{were} \mathrm{recruited} \mathrm{to} \mathrm{undergo} \mathrm{the} \mathrm{intervention} \mathrm{and}$ complete a satisfaction survey at the end of each in-person session.

Results: In total, 25 MUMSM attended one of five focus groups. Participants thought all proposed intervention topics were important and could aid in reducing sexual risk behaviors among MUMSM. However, the neurocognitive effects of methamphetamine were reported to be a barrier to practicing safer sex, condom use negotiation or HIV status disclosure. Fifteen (94\%) of 16 participants completed all 6 sessions and the satisfaction survey. On average, participants felt the intervention was useful for MUMSM, made them contemplate and move toward behavior change, and would recommend the program to their peers.

Lessons Learned: Based on our formative research, we revised the ARM-U intervention to emphasize pre-planning to avoid combining methamphetamine use and sex or develop strategies to avoid sex risk following methamphetamine use. We also increased emphasis on referrals for care and other requested services. Future efficacy trials are needed to evaluate the intervention's ability to reduce HIV-associated risk behaviors.

Keywords: HIV, injection drug use, MSM, methamphetamine, formative research, behavioral intervention.

\section{INTRODUCTION}

The strong association between methamphetamine use and high risk sexual practices among MSM has been well described [1-3]. However, there is a paucity of studies evaluating sexual risk reduction interventions among methamphetamine users, despite the strong links between use of this drug and high risk sexual practices in both gay

*Address correspondence to this author at the Division of Global Public Health, Department of Medicine, University of California San Diego, 9500 Gilman Drive, Mailcode 0507, La Jolla, CA 92093-0507, USA; Tel: (858) 822-3018; Fax: (858) 534-7566; E-mail: rgarfein@ucsd.edu and non-gay populations [4-8]. Renewed concerns about the spread of HIV/AIDS within MSM communities creates a pressing need for the development of sexual risk reduction interventions for both HIV-positive and HIV-negative sexually active MUMSM. Methamphetamine use has been associated with both transmission and acquisition of HIV among MSM $[2,3,9,10]$. Recent studies have also shown methamphetamine use to increase the rate of progression of HIV disease [11-14]. Reports of four major U.S. cities found $9.5 \%$ of MSM reported methamphatimine use within the past 6 months [15]. When erectile dysfunction drugs such as Viagra are combined with methamphetamine use, unprotected anal intercourse increases [16]. Methamphet- 
amine use also contributes to increased frequency of sex, greater number of sex partners, and engagement in sex marathons [2]. In addition, up to one-third of MSM report having injected drugs [17] potentially exposing them to parenterally transmitted HIV and hepatitis C virus (HCV) infections. A meta-analysis of sexual risk reduction interventions for drug users [18] suggests that drug users are diverse in terms of HIV status and substance abuse, thereby requiring interventions that can be tailored to meet the recipients' individual needs [18]. Thus, interventions for MSM are needed that address both sexual and substance use to prevent ongoing transmission of HIV.

Theory-based HIV prevention interventions have shown measurable effects on reducing risk behaviors in clinical trials, yet because most were conducted among highly select populations, the generalizability of their findings may be limited [19]. The natural extension of this research is to go beyond these "boutique" studies by combining elements of previously proven interventions and applying them to diverse groups that are at risk for both acquiring and transmitting HIV through sex and drug use. It is also important that such interventions are deliverable by agency staff members (e.g., social workers, counselors, outreach workers) in a variety of venues that reach MUMSM who may not possess advanced clinical or psychological training. Hence, we sought to develop a brief "toolbox", individuallevel, intervention that can be tailored by selecting modules to suit a client's individual risk profile and intervention needs, and can be delivered by counselors with an intermediate level of training.

\section{INTERVENTION DESIGN}

The Avoiding Risks from Methamphetamine Use (ARMU) intervention is a six-session program study utilizing a "toolbox" or tailored approach. The study is part of a multisite collaborative funded by the Center for Disease Control (CDC) to provide a simple intervention that can be implemented in the community. The primary goals of the intervention are to reduce sexual risk behavior and ultimately decrease the transmission of HIV. In addition, the intervention intends to decrease injection risk behaviors among IDUs and increase awareness of HIV transmission risk through injection drug use among non-IDU MSM. The intervention's target population is sexually active methamphetamine-using men who have sex with men (MUMSM) in San Diego. Participants are further classified into one of four strata: 1) injection drug user/HIV seropositive (IDU+/HIV+); 2) injection drug user/HIV seronegative (IDU+/HIV-); 3) non-injection drug user/ HIV seropositive (IDU-/HIV+); and 4) non-injection drug user/ HIV seronegative (IDU-/HIV-). Based on this classification and the participant's level of readiness to change, the health counselor uses a tailored approach relevant to each participant's unique risk factors. The ability to tailor this interevention to individual client needs addresses the fact that MUMSM as a group have diverse circumstances and HIV risks.

The intervention consists of six sessions; three in-person (sessions 1, 4, and 6 and three conducted by telephone (sessions 2, 3, and 5). This format was chosen because prior experience with MUMSM showed that frequent telephone

Table 1. Session Outline for the ARM-U Intervention

\begin{tabular}{|c|c|c|}
\hline Session & Delivery Mode & Content of Session \\
\hline 1 & In-person & $\begin{array}{l}\text { Baseline Assessment (ACASI) } \\
\text { Rapport building } \\
\text { Identify participant risk factors } \\
\text { Module } 1 \text { - Context of Unsafe Sex and Meth use } \\
\text { Module } 2 \text { - IDU-Related Risk of HIV and Injection Cross-Contamination Video (if applicable) } \\
\text { Module } 6 \text { - Referrals and Case Management } \\
\text { Make referrals to local service providers as needed }\end{array}$ \\
\hline 2 & Telephone & $\begin{array}{l}\text { Follow-up on client-identified goals } \\
\text { Follow-up on referrals } \\
\text { Re-establish or support continued motivation for change }\end{array}$ \\
\hline 3 & Telephone & $\begin{array}{l}\text { Follow-up on client-identified goals } \\
\text { Follow-up on referrals } \\
\text { Re-establish or support continued motivation for change }\end{array}$ \\
\hline 4 & In-person & $\begin{array}{l}\text { Module } 3 \text { - Safer Sex Negotiation } \\
\text { Module } 4 \text { - Disclosure of HIV Status (positive or negative) } \\
\text { Module } 6 \text { - Referrals and Case Management }\end{array}$ \\
\hline 5 & Telephone & $\begin{array}{l}\text { Follow-up on client-identified goals } \\
\text { Follow-up on referrals } \\
\text { Re-establish or support continued motivation for change }\end{array}$ \\
\hline 6 & In-person & $\begin{array}{l}\text { Module } 5 \text { - Enhancements of Social Supports } \\
\text { Module } 6 \text { - Referrals and Case Management }\end{array}$ \\
\hline
\end{tabular}


reminders were necessary to achieve high retention and we thought these interactions were a missed intervention opportunity. The intervention includes six modules that are delivered during the three in-person sessions. These modules were developed based on efficacious elements of previously conducted interventions among HIV-positive MUMSM (EDGE) [20], methamphetamine-using heterosexual men and women (Fast-Lane) [21], young adult HIV-negative IDUs (CIDUS III/DUIT) [22], and HIV-negative/HCVpositive IDUs (STRIVE) [23]. An outline of the sessions and content are listed in Table $\mathbf{1}$.

The intervention includes four core modules given to all clients, plus two need-based modules that are selected based on the client's HIV and injection drug using status (Fig. 1). The core modules address the context of unsafe sex, condom use, negotiation of safer sex practices and enhancement of social supports. The two need-based modules are tailored to address the client's HIV and IDU status. Intervention modules incorporate activities based on behavior change theories that have been found to be effective in prior studies, rather than testing new ones. Each session begins with motivational interviewing [24-26] followed by education, skills building, and practice activities based on social cognitive theory, and the theory of reasoned action. An innovative feature of the intervention focuses on the integration of subject-specific modules that can be selected based on client needs using a decision-tree algorithm resulting in an intervention that is applicable to a

\section{Core Modules}

\section{Module 1 - Context of Unsafe Sex and Meth Use \\ Goal: Increase consistent condom use. Develop insights into motivations and triggers for unsafe sex and drug use. Develop a risk reduction plan.}

Module 3 - Safer Sex Negotiation Goal: Increase safer sex practices and enhance negotiation skills through problem solving and negotiation exercises. Increase knowledge and self-efficacy through reflection, roleplay and skills building exercises.

\section{Module 5 - Enhancements of Social} Support

Goal: Enhance positive supports and minimize negative influences in relation to safer sex goals.

\section{Module 6 - Referrals and Case Management \\ Goal: Reflect on client-identified needs and make appropriate referrals as necessary.}

Fig. (1). Intervention session modules.

heterogeneous population accessed in a wide range of settings. A further advantage to this approach is that new modules can be developed to address additional client needs if deemed necessary.

The objective of the formative research presented here was to evaluate the feasibility and acceptability of the ARM$\mathrm{U}$ intervention among MUMSM and identify ways to improve the intervention prior to conducting a pilot test of the intervention. This paper describes the results of a formative process used to refine the intervention thorough focus groups and pre-testing among MUMSM who varied by HIV and IDU status.

\section{METHODS}

\section{Overview}

The intervention was developed through an iterative process that incorporated literature reviews, expert opinion, and feedback from stakeholders, intervention delivery agents, and members of the target population. This information was used to guide enhancement and revision of the intervention at each step in the process; the result being an intervention that is informed by all available information. In the formative phase we collaborated with the Centers for Disease Control and Prevention (CDC) and the other CDCfunded investigators to conduct literature reviews and analyzed existing data to inform the intervention's development. Analyses sought to identify sociodemographic, psychological, and behavioral differences among MUMSM

\section{$\underline{\text { Need-Based Modules }}$}

$\underline{\text { HIV Status }}$

IDU Status
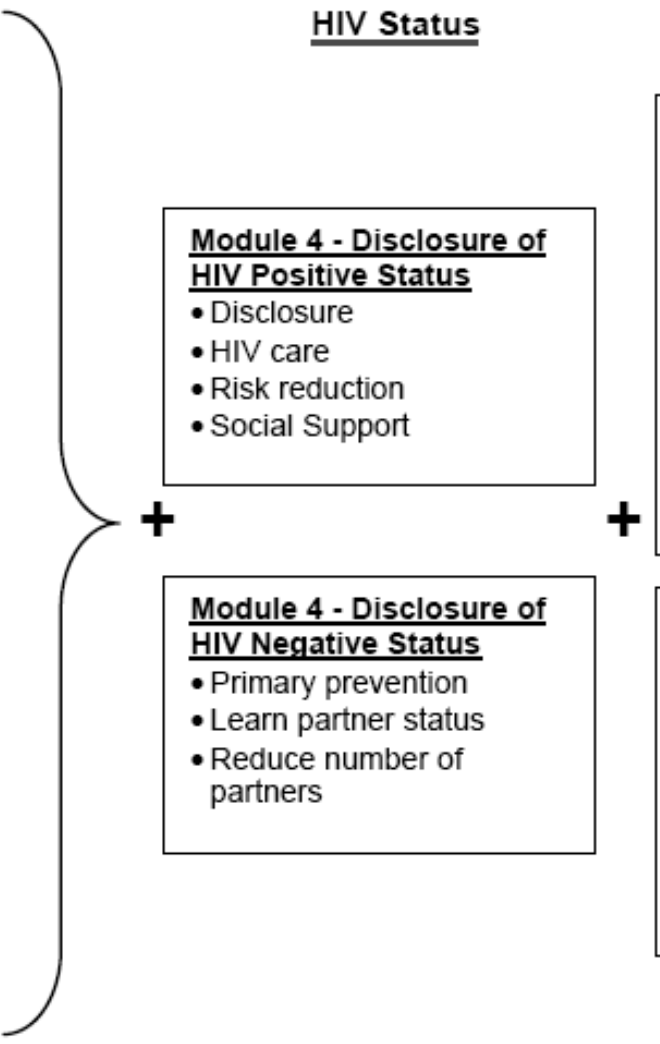

Module 2 - IDU-Related Risk of HIV and Injection CrossContamination Video

- Enhance transmission risk knowledge

- Access to clean syringes

- Negotiation to avoid sharing

- Increase self-efficacy to avoid sharing

- Drug use reduction or switch to less risky route of administration

Module 4 - Disclosure of HIV Negative Status

- Primary prevention

- Learn partner status

- Reduce number of partners
Module 2 - Non-IDU Related Risk of HIV

- Motivate decreased meth use or drug treatment entry

- Decrease risky behaviors during binge meth use

- Increase awareness that IDU sex partners are at increased risk of being HIV+ 
across the four risk strata that were used to guide the development of stratum-specific intervention modules. Focus groups were then conducted with MUMSM in each risk stratum to evaluate questions raised during the intervention development process. Using information obtained during the focus groups, the intervention was further refined and then pre-tested in its entirety with members of the target population to assess its feasibility and acceptability. Process measures and participant feedback were used to further refine the intervention materials. This study was approved by the institutional review boards at the University of California, San Diego and the CDC.

\section{Location}

This study took place in San Diego, CA where methamphetamine is a major drug of abuse and accounts for $40 \%$ of drug-related mentions in emergency departments [27]. California has a long history of methamphetamine use [28], and San Diego currently ranks third nationally in the rate of detection of methamphetamine among arrestees [29] and half of all drug treatment admissions in 2005 [30]. The sexual risk practices of methamphetamine users are a major concern for health officials in San Diego County where the drug is extremely popular and readily available [31]. Methamphetamine represents the illicit drug of choice in San Diego County with $42 \%$ of drug treatment admissions in 2007 due to methamphetamine use compared to $23 \%$ for alcohol and $8 \%$ for heroin [32]. Methamphetamine was detected among $44 \%$ of adult male arrestees during intake drug testing [33]. Nearly one-third (31\%) of the population of San Diego is Hispanic [34], enabling us to evaluate the acceptability of the intervention among Hispanic as well as non-Hispanic MSM.

\section{Participant Eligibility}

While the formative research described here was not intended to determine efficacy of the intervention for changing behavior, we applied the same inclusion and exclusion criteria in our formative research that would be used for future efficacy trials to ensure that the design was informed by the appropriate target population. Eligibility for participation in the formative phase of the study required participants to be 18 years of age or older, identify as male, report having unprotected anal intercourse with a nonprimary male partner in the past 2 months, and to have used methamphetamine at least twice in the past two months and at least once in the past 30 days. Participants also had to be able to participate in English (except for one Hispanic-only focus group) and be willing/mentally able to comply with study procedures. Identical criteria were used to enroll both focus group and intervention pre-test participants.

\section{Participant Exclusion}

Individuals were ineligible to participate if they were in or seeking drug treatment, which included residential, outpatient, support groups, 12-step or a similar program, at the time of recruitment. This exclusion was needed because it would be impossible to tease out whether changes in behavior were due to the intervention or a drug treatment program. In the intervention pre-testing phase, those participants who entered drug treatment after enrolling in the study were not excluded, so as not to penalize participants for a positive behavior change. Individuals were also ineligible if they were first diagnosed HIV-positive within the past six months or planned to move out of the county during the next five months. Additionally, the intervention was targeting MUMSM at highest risk for transmitting or acquiring HIV infection. Testing positive for HIV has been shown to be associated with significant reductions in HIVassociated risk behaviors, even in the absense of other interventions [35]. Thus, individuals who tested HIVpositive for the first time in the past six months were ineligible for this study to avoid falsely attributing behavior change to our intervention. Furthermore, focus group participants were not eligible to participate in the intervention pre-testing phase.

\section{Recruitment}

Passive and active recruitment methods were used to enroll participants for the focus groups and intervention pretesting. Flyers were posted in venues and community service agencies frequented by MSM. Display and classified advertisements were placed in periodicals and on-line resources targeting MSM. Active recruitment included street outreach in the commercial district of the local gay community. Interested individuals were instructed to call a toll-free telephone number to be prescreened and given an appointment to receive in-person eligibility screening before participating in a focus group or the intervention pre-test. Since representation by Hispanics was desired to improve generalizability of our findings, effort was made to include Hispanic MSM in all strata of this study.

\section{Focus Group Participants}

Participants were recruited for a two-hour facilitated focus group intended to refine the recruitment strategy and intervention modules. A total of five focus groups were held. The first four included MUMSM participants matched according to HIV status (+/-) and IDU experience (yes/no). The fifth group included only Hispanic MUMSM with a mix of HIV status and IDU experience. This focus group was conducted primarily in Spanish by a bilingual facilitator. Focus group participants received $\$ 20$ for their time and transportation costs. All participants provided oral and written informed consent before engaging in the focus groups.

\section{Focus Group Procedures}

The intervention design was briefly presented at the start of the focus group before initiating facilitated discussion about the intervention's acceptability and appropriateness for the target population. Focus group topics included "Is an intervention like this helpful based on your experience?"; "Is the intervention content appropriate and useful to MUMSM?"; "Is injection drug use an important concern to MUMSM?; and "What characteristics would make an appropriate counselor for this intervention?" Focus groups were led by a facilitator and an observer who took notes. The sessions were audiotaped and transcribed. Observer and facilitator brief reports were compiled into a summary report 
distributed to co-investigators for discussion. The investigators reviewed the session notes and transcripts and discussed them with the focus group facilitator and observer before using the information to make modifications to the intervention.

\section{Pre-Test Participants}

To further assess the acceptability and feasibility of the six-session intervention, MUMSM were recruited to participate in the entire intervention and provide feedback from their experiences. Four MUMSM from each of the risk strata (total 16 participants) were recruited for the intervention pre-test. At least one participant in each stratum had to be Hispanic to be sure this population was represented in the pre-test. All pre-test participants provided written informed consent prior to completing any of the pre-testing activities. Pre-test participants received \$20 after Session 1 and $\$ 15$ after each of the remaining sessions (up to $\$ 95$ total) as compensation for time and transportation costs. Compensation for in-person sessions was given immediately after the session, while compensation for telephone sessions could be picked up by appointment or at the next in-person session.

\section{Intervention Pre-Testing Procedures}

The six-session intervention was administered to members of the target population in one-on-one sessions by a single health counselor to assess the feasibility and acceptability of the content and flow of session activities. Upon completion of each in-person session (sessions 1, 4, and 6) participants completed a brief self-administered questionnaire to provide feedback on the intervention. Questions were related to each session (Fig. 1) and the intervention overall (Fig. 2). Participants were asked to rate how strongly they agreed with statements about the intervention on a scale from 1 (stongly disagree) to 10 (strongly agree). The counselor also kept session notes and recorded the duration of each session. Individual sessions were audiorecorded and reviewed by the study investigators to explore procedural elements of the intervention and monitor the counselor's adherence to the intended style of motivational interviewing and substantive content of the intervention. Similar to intervention delivery staff in health departments or community service agencies, the counselor has a masters degree in education and certification for HIV counseling and testing, but no advanced training in psychology.

\section{Intervention Description}

In session 1 the counselor administered a brief risk assessment questionnaire and conducted a reflective clientcentered discussion to initiate motivational interviewing intended to contextualize HIV risk behavior and reveal potential discrepancies between the clients' intentions and self-reported behaviors. Based on client's HIV status, the health counselor tailored a need-based module addressing disclosure of HIV serostatus, care and prevention issues, social support and reduced number of sex partners. Clients who reported IDU risk behavior also received a need-based module addressing injection-associated HIV risks and harm reduction. Participants watched a brief video (Injection Cross-Contamination) depicting how blood from one person could contaminate the syringe used by another IDU resulting in transmission of bloodborne viruses without directly sharing syringes. Non-IDUs were also shown the video if they reported having IDU sex partners to make them aware that their sex partners may be at increased risk for HIV through injection drug use. All clients also received case management and referrals to local service providers as needed.

Sessions 2, 3 and 5 were conducted by telephone and provided an opportunity to follow-up on client-identified goals, referrals, and provide empathic support encouraging continued motivation for changing risk behavior. During session 4 the counselor and client met in-person to discuss HIV disclosure and negotiation of safer sex behaviors.

In session 6, the counselor administered the content from two modules dedicated to building the client's support network and providing linkages to community services. While conducting the intervention the counselor maintained a nonjudgmental and nonconfrontational approach, guiding the client to acknowledge opportunities for reducing risk behavior while building self-efficacy. Depending on the client's needs, the counselor could resequence and tailor the content of each module, providing case managment and referrals for crisis and other situations as necessary.

\section{Community Involvement}

A Community Advisory Board consisting of community members from the target population and service agency representatives was established to assist with design of recruitment messages, materials and activities. Additionally, a Program Review Panel consisting of local health officials and community members was mandated by the funding agency to review all intervention, assessment and advertising for local acceptability. This panel also provided useful feedback on the intervention. Community service organizations, local bars and businesses that cater to the target population were instrumental in facilitating recruitment of study participants. Community partnerships were also cultivated and maintained to facilitate participant referrals to necessary service agencies.

\section{Data Analysis}

Data from focus groups consisted of transcripts and facilitators' notes, which were analyzed manually to identify themes across and within each focus group. Data from the satisfaction surveys completed by study participants following the three in-person intervention sessions were entered into a database, analyzed using descriptive statistics for ordinal data, and graphically presented to show the level of agreement with specified statements about the intervention.

\section{RESULTS}

\section{Focus Groups}

A total of 25 participants took part in one of the five focus groups between October and November 2007. The 


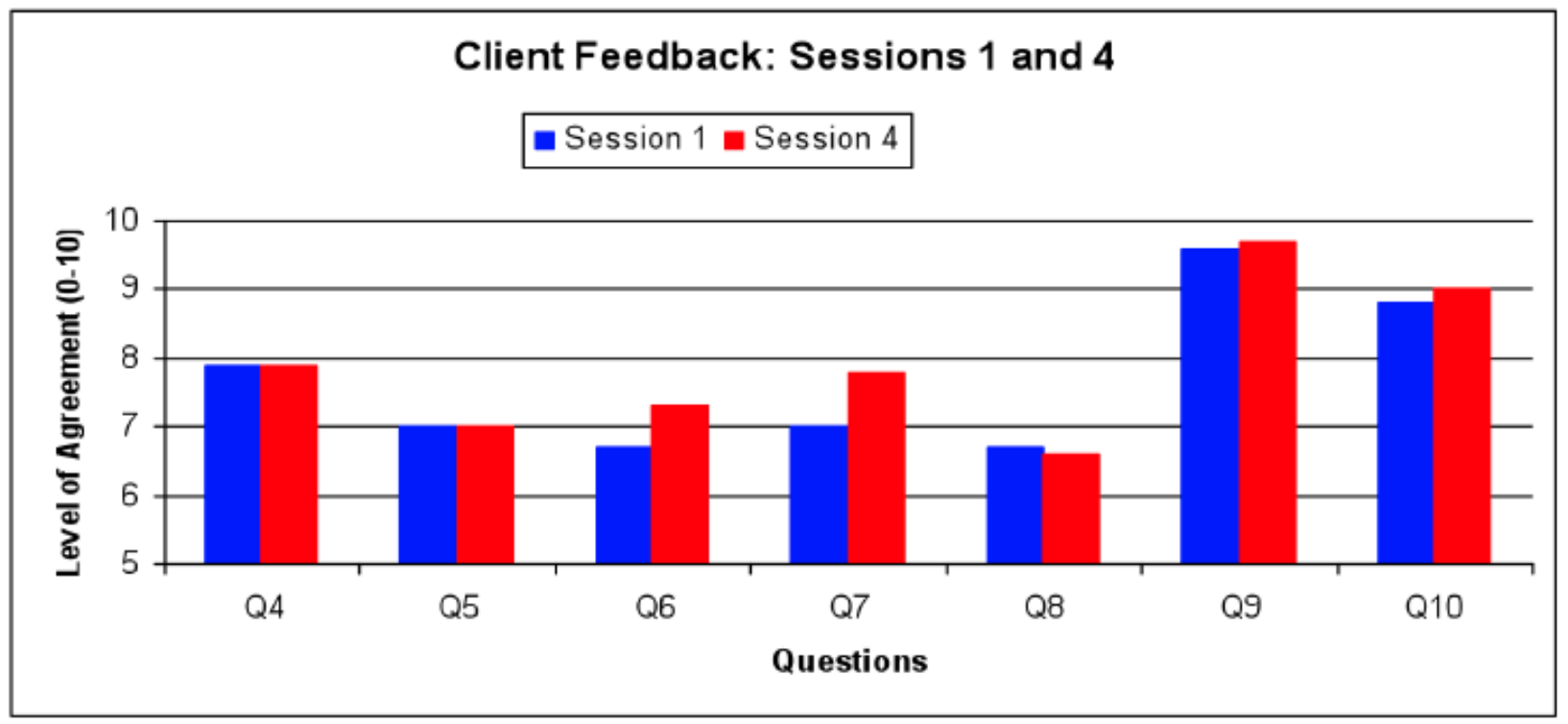

Fig. (2). Participant feedback on Sessions 1 and 4 of the ARM-U intervention.

For questions 4-10, on a scale from 1 (stongly disagree) to 10 (strongly agree), please indicate the level to which you agree with each of the following statements:

Q4: Provided me w/information about protecting my health, which I intend to use

Q5: Made me think about protecting my health in new ways

Q6: Made me think about protecting my sex partners' health in new ways

Q7: I feel more motivated to protect my own and partners' health now than before session

Q8: I feel more capable of protecting my own and partners' health now than before session

Q9: The health counselor was knowledgeable about session topics

Q10: The health counselor could easily relate to my situation and lifestyle

mean age of the participants was 44 years (range 24-58 years); 9 (36\%) were white, 7 (28\%) were Black, 9 (36\%) were Hispanic, 1 (4\%) was Asian, and 1 (4\%) was mixed race. Attempts to recruit Hispanic IDUs were only successful for the Hispanic and non-IDU focus groups. Overall, there were no major differences in the themes identified across the 5 focus groups. Specific themes that emerged from the focus groups are described below.

\section{Is Methamphetamine Use an Important Factor in HIV Risk Reduction Among MUMSM?}

For the majority of participants, the relationship between methamphetamine and sex was tightly linked. As one participant put it "I personally think that they are synonymous....sex and crystal [go] hand in hand". Another stated: "sex and meth were completely fused together". When participants' were asked specifically about unsafe (i.e. unprotected) sex they explained clearly that upon administration of methamphetamine, the probability of unsafe sex increases initially and then as fatigue sets in the probability is reduced. One participant stated:

"the thought [of safe sex] may be there prior to the injection or the smoking or the inhaling, but once you are induced and under the influence you are so vulnerable you have almost no protection for yourself."
Many participants also referred to the "meth state of mind" in which known risky behaviors would be perceived less or non-risky when under the influence of methamphetamine. These statements support the development and implementation of an intervention to address safer sexual and drug use behavior practices within the perceived inseparable relationship between methamphetamine and sex.

When asked about willingness to change and motivation to change participants commented in a variety of ways. Many believed that "[willingness] is everything" and without it a person will not change. One participant described it as:

"you don't see nothing and you don't want to hear nothing. And that's what drugs does to people. It puts a blindfold and earplugs in your ears. You don't see nothing. You don't hear nothing - on with the game."

Furthermore, many participants acknowledged that motivation to change is important, but that motivation alone is not enough:

"Before the high you are straight. Before the high you are not going to have sex. Before the high you are only going to do this, that, or the other thing. But, baby, once the rush is on..."

Also, for some participants an emphasis on motivation for reducing risk behaviors did not appear to be received 
well. It may be that for some, gauging motivation for safer practices is not compatible with methamphetamine use. Thus, it may be more appropriate to focus on what in the individual's current environment could be changed or altered to reduce risk behaviors. Reliance on changing cognitions leaves the intervention liable to the known negative neurocognitive consequences related to methamphetamine use and HIV-infection, and thus potential loss of the intervention effects.

\section{Would an Intervention Like ARM-U be Appropriate for IDU and Non-IDU MSM?}

One concern in developing an intervention for both injecting and non-injecting MUMSM was that the groups might differ vastly. Thus, participants were asked how injecting and non-injecting methamphetamine users differed. As one participant put it, the method of administration was tied to how the users behaved:

\begin{abstract}
"[Methamphetamine] smokers sit around and talk about it a lot. They sit around and they talk and talk and talk and talk and talk...And then you have people that snort it. They're like, you know, they have their little projects that they work on. Sometimes they've got two or three or four different little projects that they're working on, you know, but they tend to usually get them haphazardly done, you know, and then go on to the next one. Whereas like, the IV users, they do it and they want dick or pussy or whatever it is that they're interested in."
\end{abstract}

While some participants look down on IDUs, their opinions frequently changed depending on their circumstance: "My personal experience was that the day before I put a needle in my arm I thought that junkies were the scuz of the earth." However, rather than segregation by preferred method of use, there appears to be a continuum among users that suggests interaction between these groups.

"I started snorting it and it didn't work for me anymore, and then the smoking came out. So I started smoking it and when the smoking didn't work for me I started slamming it, so it's basically you get to a certain level, for me, I can't speak for other people, you get to a different level of your getting high, and then when you don't achieve that high anymore you want to go to something different, especially with meth, and when you go from snorting to smoking it's a whole different thing, and then from smoking to slamming it's a whole different world."

\section{Is the Intervention Content Appropriate and Useful to MUMSM?}

Although half of the focus groups participants had never injected drugs, most reported knowing people who had. There was also general interest in transmission through injection drug use and many had questions about whether hepatitis could be sexually transmitted. Therefore, the topic of injection drug use had relevance even among MUMSM who were not themselves IDUs. Furthermore, although not stated explicitly in any of the focus groups, many of the participants implied that they were information brokers and/or models of behavior within their social network. Thus, this intervention, like needle exchange programs, has the potential for both primary and secondary participants. This supports showing the Injection Cross-Contamination video to all participants regardless of injection status and might support overlaps in the tailored sessions for each risk strata.

The negotiation of condom use for most participants was viewed as not feasible or non-existent in the context of methamphetamine use. One participant stated:

\section{'Negotiation doesn't matter if it is something I wanted to do prior to me using. Once I started using the rules change. The rules change."}

This was echoed by other participants of both HIVpositive and HIV-negative status. In fact, it appeared that for HIV-negative participants, the act of using a condom meant you were HIV-positive; however, for HIV-positive participants using a condom meant that you were HIVnegative. Consequently, the HIV-negative participant did not want to wear a condom and be viewed as HIV-positive whereas, the HIV-positive participant didn't want to use a condom because it was assumed if you didn't use a condom you were already HIV-positive. Thus, the challenge is how to break the link between condom use and perceived HIV status. Basically, condom use does not appear to indicate an individual's current HIV status and HIV status does not indicate condom use within the context of methamphetamine use. This point is summed up by two participants' statements:

"I will do what you want as long as I can get high" and "when I am using I am not really giving a rat's ass about anybody else but getting high".

Disclosure of HIV status appeared to be context specific. Many participants implied a "don't ask, don't tell" type of policy when at bathhouses and parties. However, others stated that HIV status made a difference. If an individual is HIV-negative they are more likely to disclose than an HIVpositive individual. However, this was not the consensus among the groups and several participants mentioned that they take a more defensive approach:

"I sort of act like everyone has [HIV] to begin with, so it doesn't matter what they tell me whether they say they're negative or positive. I act the same way, because I don't want to get it."

This approach is supported by participants' experiences with dishonesty about disclosure. One participant offered this:

"you state whether you are HIV-positive or negative, but a lot of people lie, which is sad. And then there's these kids or people that they call them bug chasers - they want to get 
infected just to not worry about cleanliness you know, and then there's the HIV-positive guys that want to infect negative, you know. They look for the bug chaser."

Referrals, and to a lesser extent social supports, emerged as important themes from the focus groups that should be emphasized in the intervention. For example, one participant emphasized the inequity in accesses to referrals and resources:

"If you went to prison or if you went to jail they'll link you right along, you know, or if you're positive and you're cool you've got all the resources, but if you're negative you get really no linkage between the support groups out there."

Several participants raised concerns about the quality of social support available to them. One participant offered his experience:

"I made the huge mistake of thinking I could turn to family and tell them that I was slamming dope. Big mistake.....you know they'd never trust me again now. It's like I'm a slammer - you know, all the stereotypes that [they] saw from the TV - now that's what I am, so you know I thought, because my life was going down the toilet so I reached out to my family and when I told them what I was doing, I thought that would shock them into getting me some help, like them doing something physically by going out and looking for some help for me to get me there because I couldn't do it on my own and it obviously didn't happen."

Repairing this perceived and/or real deficit in the quality of social support may be beyond the scope of a brief individual-level intervention such as ARM-U. However, referrals to self-help and/or other community groups could increase the quality of social support and eventually assist in adopting safer sexual and drug use practices.

\section{What Characteristics Would Make an Appropriate Counselor for this Intervention?}

Participants gave feedback on what would make a "good counselor". Several characteristics were discussed ranging from sociodemographics to personal experience with drugs and HIV. Participants, including those in the Hispanic focus group, almost universally said they preferred male counselors. Similarly, participants felt that it was important that the counselor be an MSM as described by one participant:

"I'd rather talk to somebody that's been dealing with my issue. I would rather do that and I know that first-hand, so I would feel comfortable. It's just like I'd rather speak to somebody gay about gay things when someone's gay. I'm not gonna tell some straight."
There was consensus among most of the participants that it was important for the counselor to have relevant personal experience. Experience was defined in terms of real life events and practices. This was illustrated by one participant who said:

"[The counselors] need to have common experiences."

A minority of participants mentioned that it was also important for the counselor to have advanced education in counseling. For example, one participant stated: "He doesn't have to have the experience. He has to have knowledge and education background and a history of drug use is not necessary." Thus, the personal experience and education of the counselor appears to be relevant to how participants will relate to them; however, further research is needed to determine the impact of counselor self-disclosure on the efficacy of individual-level behavioral interventions.

\section{Intervention Pre-Testing}

Upon completion of the focus groups and revision of the intervention based on focus group findings, the intervention was implemented in its entirety with all new volunteers. Between September 2007 and January 2008, a total of 16 participants were consented and took part in the first intervention session and 15 (94\%) completed all six sessions. Among the pre-test participants, $9(56 \%)$ were white, 3 $(19 \%)$ were Black, 3 (19\%) were Hispanic, and 1 (6\%) was Asian. Although we attempted to recruit at least one Hispanic MUMSM for each risk stratum, none of the Hispanic participants were IDU, suggesting that this population is small in San Diego or more hidden than nonHispanic IDU. The mean age of the pre-test group was 44 years old (range 35-58 years old). Although the intervention could be completed in three weeks, it took on average 39 days (range 21-62 days) to complete the six sessions, due to rescheduling missed appointments.

Immediately following the in-person sessions (sessions 1, 4, and 6), all participants completed an intervention feedback questionnaire. Participants unanimously agreed (all scores $\geq 5$ ) with each of the statements about the intervention after sessions 1 and 4 (Fig. 2). Notably, the level of agreement with the statements "[the intervention] made me think about protecting my sex partners' health in new ways" and "I feel more motivated to protect my own and partners' health now than before the session," increased between sessions 1 and 4, albeit not statistically significant. This finding suggests that the intervention content regarding negotiation and HIV status disclosure with sex partners presented during session 4 had the desired effect. Regarding characteristics of the health counselor, participants strongly agreed that the counselor was knowledgeable about the intervention topics and could easily relate to the participants.

Following session 6 , participants were asked to evaluate the intervention overall. Participants unanimously agreed, many strongly, that the intervention motivated them to think about their sexual behavior, methamphetamine use, and life in general (Fig. 3). All participants strongly agreed with the statement, "I would recommend this program to other men 


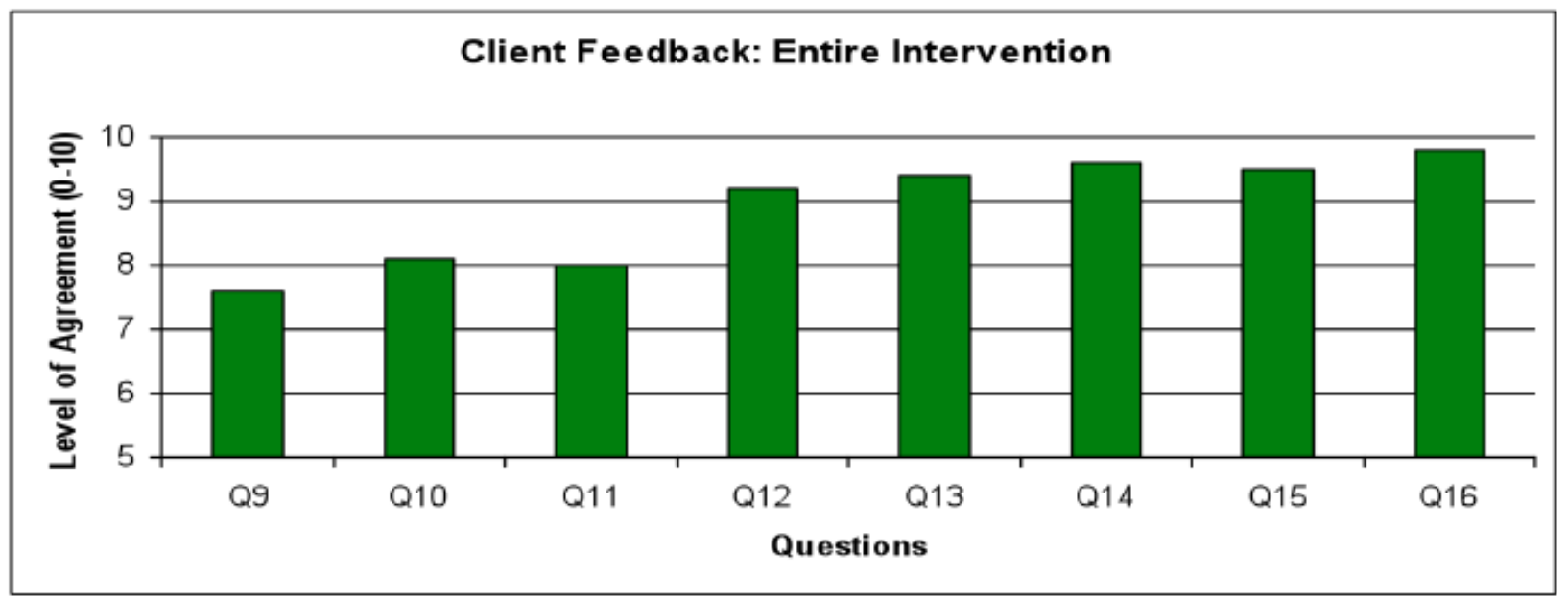

Fig. (3). Participant overall feedback on the ARM-U intervention.

For questions 9-16, on a scale from 1 (stongly disagree) to 10 (strongly agree), please indicate the level to which you agree with each of the following statements:

Q9: Overall, I learned a lot form this program

Q10: Overall, the program motivated me to think about my sexual behavior

Q11: Overall, the program motivated me to think about my Meth use

Q12: The program motivated me to make positive changes in my life

Q13: I would recommend this program to other men like me

Q14: The session health counselor was knowledgeable

Q15: The session health counselor was supportive

Q16: The session health counselor was respectful of me

like me." The health counselor made a positive impression on the participants as they strongly agreed that he was knowledgeable, supportive and respectful to the participants. No differences were observed in the levels of agreement between participants in the four risk strata.

In the original intervention design, modules 2,3 and 5 were intended to be delivered during the telephone sessions. However, it was quickly discovered that participants frequently had other concerns that took precedence over the counselor's attempts to deliver the module content. Therefore, the modules were moved to the three in-person sessions and the telephone sessions were used to follow-up on client-identified goals and referrals, and to support continued motivation for change. While participants were not specifically surveyed regarding their impressions of the three telephone sessions, all but one participant completed all of the telephone sessions suggesting that these sessions were not prohibitive for participants. According to the counselor's notes, telephone sessions were productive and provided the participants an opportunity to "check-in" with the counselor and discuss issues that came up since the last in-person session.

\section{DISCUSSION}

Through the formative research process, we identified strengths and weaknesses in the ARM-U intervention. Based on the focus groups, we determined that participants agreed that the planned content of all modules was important and could help reduce sexual risk behaviors among MUMSM. These groups also validated our assumption that injection drug use had a significant presence among MUMSM regardless of whether the participant was an IDU himself and, thus, all clients should receive some information about risks from injection drug use (e.g., Injection CrossContamination). One troubling finding was that once under the influence of methamphetamine, MUMSM reported not being able to control impulses and would subsequently engage in unsafe practices. This finding has important implications for interventions that take a harm reduction rather than an abstinence approach to methamphetamine use. Although some behavioral interventions have been shown to reduce sexual risk behaviors among MSM [20] and heterosexuals [21] in the context of methamphetamine use, interventions that reduce methamphetamine use among MUMSM may be a necessary complement to harm reduction [36]. In response, the ARM-U intervention was revised to place greater emphasis on pre-planning and abstaining from methamphetamine use, rather than trying to control their situation after using methamphetamine.

We also established closer linkages with treatment programs that serve methaphetamine users to help facilitate referrals for treatment. As expected, participants frequently expressed the need for more resources to help them fulfill their desires to reduce their risk of acquiring or transmitting HIV or other infections. Thus, all intervention sessions were 
revised to include time to review the need for health, social, substance abuse, and other services with the participant.

An important observation was that condom use was often linked to misperceptions about HIV status among MSM. Consequently, the ARM-U intervention was revised to include greater discussion about how MSM should not presume they or their partners can predict each others HIV status based on whether or not condoms are introduced into a sexual event. In addition, greater emphasis will be placed on HIV status disclosure and condom use negotiation skills.

A weakness of this intervention, as with most other interventions, is that its effectiveness may depend on the counselor's ability to engage and establish rapport with the client. Efficacy trials that take counselor effect into account in their design are needed to determine the importance of this factor. In addition, prior studies of brief interventions such as ARM-U suggest that the effects of behavioral interventions might be transient $[37,38]$. Thus, future evaluation of ARMU should follow participants for six months or more postintervention to determine whether booster sessions are needed to achieve long-lasting behavior change. Although every attempt was made to recruit at least one Hispanic MSM in each of the four risk strata, we were unable to enroll Hispanic IDUs for the pre-test. Consequently, we can only speculate about how the intervention will be received by this group.

Through this study, we found that MUMSM in all stata of HIV and IDU status can be recruited and retained for behavioral intervention trials that tailor the intervention to each individual's status. The six-session ARM-U intervention was feasible and acceptable to MUMSM in our formative study. If found to be efficacious and cost-effective in future trials, this design would provide a broadly applicable intervention for MUMSM and would be an excellent complement to HIV counseling and testing programs, such as those already being delivered by providers with varying degrees of training in a wide variety of settings.

\section{ACKNOWLEDGEMENTS}

We are grateful for the collaborative feedback on our intervention from the CDC (Gordon Mansergh, Mahnaz Charania), University of California Los Angeles (Cathy Reback), RTI (Bill Zule), and Columbia University (Elwin $\mathrm{Wu}$ ). Ronald Stall and Jim Zians provided consultation on the intervention design. We also acknowledge the undaunted efforts of the ARM-U team, which included David Rodriguez, Michele Velasquez, Nicholas Aldridge, and Philippe Duhaime, as well as Jill Kunkel at the Antiviral Research Center for her logistical assistance conducting this study.

This study was funded through a cooperative agreement (UR6 PS000306) with the Centers for Disease Control and Prevention, National Center for HIV, Hepatitis, STD and Tuberculosis Prevention.

\section{REFERENCES}

[1] Reback CJ. The social construction of a gay drug: Methamphetamine use among gay and bisexual males in Los Angeles. Executive Summary. City of Los Angeles, USA: AIDS Coordinator 1997.
[2] Semple SJ, Patterson TL, Grant I. Motivations associated with methamphetamine use among HIV+ men who have sex with men. J Subst Abuse Treat 2002; 22(3): 149-56.

[3] Colfax G, Coates TJ, Husnik MJ, et al. Longitudinal patterns of methamphetamine, popper (Amyl Nitrite), and cocaine use and high-risk sexual behavior among a cohort of San Francisco men who have sex with men. J Urban Health 2005; 82(Suppl 1): i62-72.

[4] Forsch D, Shoptaw S, Huber A, Rawson R, Ling W. Sexual HIV risk among gay and bisexual male methamphetamine abusers. J Subst Abuse Treat 1996; 13(6): 483-486.

[5] Gorman M. A tale of two epidemics: HIV and stimulant use. FOCUS 1998; 13(4): 1-8.

[6] Hando J, Hall W. HIV risk-taking behavior among amphetamine users in Sydney, Australia. Addiction 1994; 89: 79-80.

[7] Klee $\mathrm{H}$. A new target for behavioral research-amphetamine misuse. Br J Addict 1992; 87: 439-46.

[8] Colfax G, Shoptaw S. The methamphetamine epidemic: implications for HIV prevention and treatment. Curr HIV/AIDS Rep 2005; 2(4): 194-9.

[9] Drumright LN, Patterson TL, Strathdee SA. Club drugs as causal risk factors for HIV acquisition among MSM: a review. Subst Use Misuse 2006; 41(10-12): 1551-601.

[10] Yeon P, Albrecht H. Crystal methamphetamine and HIV/AIDS AIDS Clin Care 2008; 20(2): 2-4.

[11] Ellis R, Childers M, Cherner M, Lazzaretto D. Increased human immunodeficiency virus loads in active methamphetamine users are explained by reduced effectiveness of antiretroviral therapy. J Infect Dis 2003, 188(12): 1820-6.

[12] Mahajan SD, Aalinkeel R, Sykes DE, et al. Methamphetamine alters blood brain barrier permeability via the modulation of tight junction expression: implication for HIV-1 neuropathogenesis in the context of drug abuse. Brain Res 2008; 1203: 133-48.

[13] Potula R, Persidsky Y. Adding fuel to the fire: Methamphetamine enhances HIV infection. Am J Pathol 2008; 172: 1467-70.

[14] Tallóczy Z, Martinez J, Joset D, et al. Methamphetamine inhibits antigen processing, presentation, and phagocytosis. PLoS Pathogens 2008; 5(2): 0001-11.

[15] Stall R, Paul JP, Greenwood G, et al. Alcohol use, drug use and alcohol-related problems among men who have sex with men: the Urban Men's Health Study. Addiction 2001; 96: 1589-601.

[16] Mansergh G, Purcell DW, Stall R, et al. CDC consultation on methamphetamine use and sexual risk behavior for HIV/STD infection: Summary and suggestions. Public Health Rep 2006; 121(2): 127-32.

[17] Semple SJ, Patterson TL, Grant I. A comparison of injection and non-injection methamphetamine-using HIV positive men who have sex with men. Drug Alcohol Depend 2004; 76(2): 203-12.

[18] Semaan S, Des Jarlais DC, Sogolow E, et al. A meta-analysis of the effect of HIV prevention interventions on the sex behaviors of drug users in the United States. J Acquir Immune Defic Syndr 2002; 30: S73-93.

[19] Des Jarlais DC, Semaan S. HIV prevention research: cumulative knowledge or accumulating studies? An introduction to the HIV/AIDS Prevention Research Synthesis Project supplement. J Acquir Immune Defic Syndr 2002; 30: S1-7.

[20] Mausbach BT, Semple SJ, Strathdee SA, Zians J, Patterson TL. Efficacy of a behavioral intervention for increasing safer sex behaviors in HIV-positive MSM methamphetamine users: Results from the EDGE study. Drug Alcohol Depend 2006; 87(2-3): 24957.

[21] Mausbach B, Semple S, Strathdee S, Zians J, Patterson T. Efficacy of a behavioral intervention for increasing safer sex behaviors in HIV-negative, heterosexual methamphetamine users: Results from the Fast-Lane Study. Ann Behav Med 2007; 34(3): 263-74.

[22] Garfein RS, Golub ET, Greenberg A, et al. A peer-education intervention to reduce injection risk behaviors for HIV and $\mathrm{HCV}$ infection in young injection drug users. AIDS 2007; 21: 1923-32.

[23] Latka MH, Hagan H, Kapadia F, et al. A randomized intervention trial to reduce the lending of used injection equipment among injection drug users infected with Hepatitis C. Am J Public Health 2008; 98: 853-61.

[24] Johnson WD, Holtgrave DR, McClellan WM, Flanders WD, Hill AN, Goodman M. HIV intervention research for men who have sex with men: A 7-year update. AIDS Educ Prev 2005; 17(6): 568-89.

[25] Koblin B. Effects of a behavioural intervention to reduce acquisition of HIV infection among men who have sex with men: 
The EXPLORE randomised controlled study. Lancet 2004; 364(9428): 41-50.

[26] Strathdee SA, Patterson TL. Behavioral interventions for HIV positive and HCV positive drug users. AIDS Behav 2006; 10: 11530 .

[27] Pollini R, Strathdee S. Indicators of methamphetamine use and abuse in San Diego County, California: 2001-2005. J Psychoactive Drugs 2007; (Suppl 4): 319-25.

[28] SAMHSA. Methamphetamine use, abuse, and dependence: 2002 , 2003, and 2004. The NSDUH Report, September 16, 2005. Available from: http: //www.oas.samhsa.gov/2k5/meth/meth.htm [last accessed on 10/07/09].

[29] SAMHSA. Trends in methamphetamine/amphetamine admissions to treatment: 1993-2003. The DASIS Report, Issue 9, 2006; Available from: http://www.oas.samhsa.gov./2k6/methTx/methTX.htm [Last accessed on $10 / 07 / 09]$.

[30] National Institute on Drug Abuse. Epidemiologic Trends in Drug Abuse: Proceedings of the Community Epidemiology Work Group, Bethesda, MD: National Institutes of Health, January 2006; vol. II: Available from: http://www.drugabuse.gov/PDF/CEWG/Vol2_60 6.pdf [last accessed on 3/10/10].

[31] National Institute on Drug Abuse. Epidemiologic Trends in Drug Abuse: Vol. II., Proceedings of the Community Epidemiology Work Group, January 2006. NIH Pub. No. 06-5880, Bethesda, MD: National Institutes of Health. Available from: http://www. drugabuse.gov/PDF/CEWG/Vol2_606.pdf [last accessed on $3 / 10 / 10]$.
[32] National Institute on Drug Abuse. Epidemiologic Trends in Drug Abuse: Vol. I., Proceedings of the Community Epidemiology Work Group, June 2008. NIH Pub. No. 09-6421, Bethesda, MD: National Institutes of Health. Available from: http://www.drugabuse.gov/ PDF/CEWG/CEWGJune08vol\%201_web.pdf [last accessed on $3 / 10 / 10]$.

[33] Burke C. Methamphetamine use among San Diego County arrestees. J Psychoactive Drugs 2007; (Suppl 4): 337-45.

[34] U.S. Census Bureau State \& County Quick Facts, 2008. Available from: http: //quickfacts.census.gov/qfd/states/06/06073.html [last accessed on 11/7/09].

[35] Marks G, Crepaz N, Senterfitt J, Janssen R. Meta-analysis of highrisk sexual behavior in persons aware and unaware they are infected with HIV in the United States. J Acquir Immune Defic Syndr 2005; 39(4): 446-53.

[36] Shoptaw S, Reback CJ, Peck JA, et al. Behavioral treatmen approaches for methamphetamine dependence and HIV-related sexual risk behaviors among urban gay and bisexual men. Drug Alcohol Depend 2005; 78(2): 125-34.

[37] Parsons JT, Golub SA, Rosof E, Holder C. Motivational interviewing and cognitive-behavioral intervention to improve HIV medication adherence among hazardous drinkers: a randomized controlled trial. J Acquir Immune Defic Syndr 2007; 46(4): 443-50.

[38] Patterson TL, Shaw WS, Semple SJ. Reducing the sexual risk behaviors of HIV+ individuals: outcome of a randomized controlled trial. Ann Behav Med 2003; 25(2): 137-45.

(C) Garfein et al.; Licensee Bentham Open.

This is an open access article licensed under the terms of the Creative Commons Attribution Non-Commercial License (http://creativecommons.org/licenses/by-nc/ 3.0/) which permits unrestricted, non-commercial use, distribution and reproduction in any medium, provided the work is properly cited. 